\title{
UNIVERSITYOF
}

FORWARD

THINKING

WESTMINSTER ${ }^{\text {网 }}$

WestminsterResearch

http://www.westminster.ac.uk/westminsterresearch

\section{Iris Image Recognition using Optimized Kohonen Self Organizing}

Neural Network

Jenkin Winston, J., Jude Hemanth, D., Angelopoulou, A. and Kapetanios, E.

This is a copy of the author's accepted version of a paper subsequently published in the proceedings of the 9th International Conference on Imaging for Crime Detection and Prevention, 16 - 18 Dec 2019 . doi:10.1049/cp.2019.1171.

The final published version will be available online at:

https://dx.doi.org/10.1049/cp.2019.1171

(C) 2019 IEEE . Personal use of this material is permitted. Permission from IEEE must be obtained for all other uses, in any current or future media, including reprinting/republishing this material for advertising or promotional purposes, creating new collective works, for resale or redistribution to servers or lists, or reuse of any copyrighted component of this work in other works.

The WestminsterResearch online digital archive at the University of Westminster aims to make the research output of the University available to a wider audience. Copyright and Moral Rights remain with the authors and/or copyright owners. 


\title{
Iris Image Recognition using Optimized Kohonen Self Organizing Neural Network
}

\author{
J. Jenkin Winston', D. Jude Hemanth ${ }^{1,}{ }^{*}$, A. Angelopoulou ${ }^{2}$, E. Kapetanios ${ }^{2}$ \\ ${ }^{1}$ Department of ECE, Karunya Institute of Technology and Sciences, Coimbatore, India \\ ${ }^{2}$ Cognitive Computing Research Lab, University of Westminster, UK \\ *Corresponding Author e-mail: judehemanth@karunya.edu
}

Keywords: Iris, Biometrics, Machine learning, Neural network, Optimization.

\begin{abstract}
The pursuit to develop an effective people management system has widened over the years to manage the enormous increase in population. Any management system includes identification, verification and recognition stages. Iris recognition has become notable biometrics to support the management system due to its versatility and non-invasive approach. These systems help to identify the individual with the texture information distributed around the iris region. Many classification algorithms are available to help in iris recognition. But those are very sophisticated and require heavy computation. In this paper, an improved Kohonen selforganizing neural network (KSONN) is used to boost the performance of existing KSONN. This improvement is brought by the introduction of optimization technique into the learning phase of the KSONN. The proposed method shows improved accuracy of the recognition. Moreover, it also reduces the iterations required to train the network. From the experimental results, it is observed that the proposed method achieves a maximum accuracy of $98 \%$ in 85 iterations.
\end{abstract}

\section{Introduction}

Authentication through recognition is the basic step of any management system. Usually, recognition is either done by token-based systems or knowledge based systems. Smart cards or ID cards used in the token-based method can be misused or it can get lost. Moreover, the PIN and passwords used in knowledge-based systems can make the user difficult to remember them. To make this process simple we employ biometric systems that can recognize individuals using biometric traits. These biometric traits can be either physiological or the behavioural attribute of the individuals. Out of many traits, the iris is more peculiar because it is extremely unique even between twins. Moreover, the iris is made of layers made of collagenous muscles and pigmented epithelial cells. This provides iris a rich texture [1,2]. Hence, the iris is chosen as a biometric trait in this paper.

Figure 1 shows the generic block diagram of the iris recognition system. It consists of iris image pre-processing, feature extraction and classifier. The iris images downloaded from the database are prepossessed. Pre-processing involves enhancing the iris image, and the localizing the iris region and normalizing it into a fixed block size. The feature extraction block involves capturing the best descriptors from the normalized iris region. These features are used by classifiers in training and testing the input iris images.

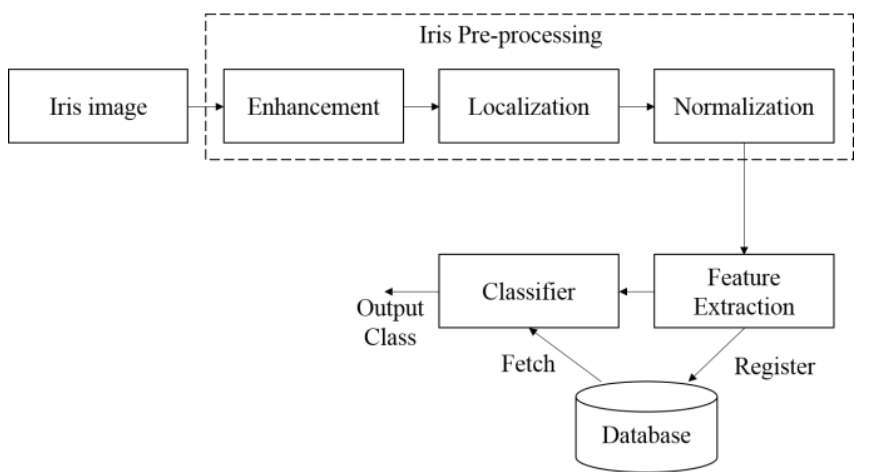

Figure 1. Generic block diagram of iris recognition system

There are many classifiers proposed by researchers for iris recognition. Few related works are discussed below. Multiple classifiers namely Linear classifier, Fisher classifier, Tree classifier, Neighbour classifier, Parzen classifier, and SVM classifier are employed in this paper [3] to improve the accuracy of iris recognition. Each classifier is good at reading different feature space. Genetic algorithm along with circular symmetric filters are used to choose the best classifiers and fuse the results for improving the accuracy. But the increased accuracy comes at the cost of increased computation.

In [4], the author has suggested a hybrid approach that engages three levels of fusion such as decision fusion, score fusion, and feature fusion. Feature reduction is done using principle component analysis. The final decision is made with the fusion of outputs from discrete hidden Markov modelbased classifier applied to the left iris, right iris, feature fused left-right iris and the log-likelihood ratio of feature fused leftright iris. The multiple classifiers utilized in the method increases the learning and testing time of the system.

The authors in [5], have adaptive bloom filters in recognizing the iris pattern. Bloom filters generate templates that are rotation invariant. This filter based classifier does not suffer any alignment problem during the comparison. The compression on iris templates generated from Bloom filter by 20 to $40 \%$ yields a reasonable accuracy. This increases the speed of comparison. The reason for this is that the reduced template reduces the computation required by the recognition system. 
A repetitive partitioning method is recommended for iris recognition by V.V.S. Tallapragada et al. in [6]. Hard thresholds are applied to segment the iris region. From the segmented iris region, Tamura features are computed and compared using a decision tree. The decision tree optimizes the cost function to find the class of the test iris image. With the limited features, the accuracy attained by this method is very poor for practical applications.

To increase the rapidity and robustness in iris recognition, Y. Si et al. [7] have proposed three fold method. Eyelashes are detected using directional filters to avoid misclassification. Multidirectional data fusion is done to nullify the edge effect due to improper segmentation. To accelerate the searching process, an iris indexing method is proposed. This enhances the performance of the iris recognition system.

A discriminative dictionary learning framework acquired from K-SVD is hypothesized by Y. Song et al. [8]. Initially, a sparse error correction mode is adopted to improve the recognition rate of iris occluded by eyelashes. The introduction of a compact dictionary into the learning phase further improves the performance of the system.

A structure for iris recognition using support vector machine and hidden Markov model is proposed by V.V.S. Tallapragada et al. in [9]. Using Kernel Fisher Analysis iris features are mapped into a higher dimensional space. Through a Radial Basis Function and annealing optimization the features are partitioned into suitable classes in reference to support vector machine.

For the statistically unique iris patterns a multi-dimensional neural network is proposed by R.M. Farouk et al. in [10]. It has eight neurons in the input layer, twelve neurons in the hidden layer and one neuron output layer. Based on the error signal, the network passes the information backward to update the weights. Experimental values show better matching capabilities. But, much exploration on the speed of recognition can be also made to improve performance.

A non-parametric supervised learning algorithm named KNN classifier is imparted for iris recognition by D. Choudhary et al. in [11]. It measures the closeness of the test image to the database by computing Euclidean distance. The decision is made on the major voting by the surround $\mathrm{K}$ data points. The real challenge in this method is to determine the value of $K$, because as the $\mathrm{K}$ increases the computation complexity of the algorithm also increases.

A neural network approach using Learning Vector Quantization (LVQ) is propounded for classifying iris by authors in [12]. This method has the supremacy of statistical and neural network characteristics to build the recognition system. It consists of an input layer and output layer. The output assigned based on the voting of majority classifiers. Along with the local binary pattern, histogram features, the LVQ classifier performs better.

This paper provides an understanding of the different stages involved in iris recognition. In this work, modification in the operation of Kohonen Self-Organizing Neural Network is introduced to enhance the performance of classification. Section 2 briefs about the proposed methodology. In Section 3 , the conventional KSONN is elaborated. In section 4, optimization-based KSONN using Gravity Search Algorithm and Particle Swarm Optimization is discussed. The results obtained from the proposed methodology are examined in section 5. Atlast, the conclusions are reviewed in section 6 .

\section{Proposed methodology}

In this work, a hybrid Kohonen Self-Organizing Neural Network is proposed as a classifier for iris recognition. Figure 2 describes the outline of the method done in this work. A physics-based optimization, human-based optimization and a swarm intelligence based optimization technique are employed into the network architecture to improve the learning process and increase accuracy. This paper highlights the benefits of introducing an optimization technique into the learning phase of the KSONN network. The images in the iris database suffer low contrast because of non-uniform illumination. This is corrected using Contrast Limited Histogram Equalization in the enhancement stage. Later, the iris region is localized using Circular Hough Transform. This helps the iris region separated from the surrounding tissues. Next, the circular iris region is mapped into a rectangular block of fixed size using normalization. In the upcoming stage, the statistical features are computed from the moment space of the normalized iris image. This serves as the input for the KSONN network for classification. The idea of this paper is to compare the results of conventional KSONN with the hybrid KSONN proposed using optimization techniques.

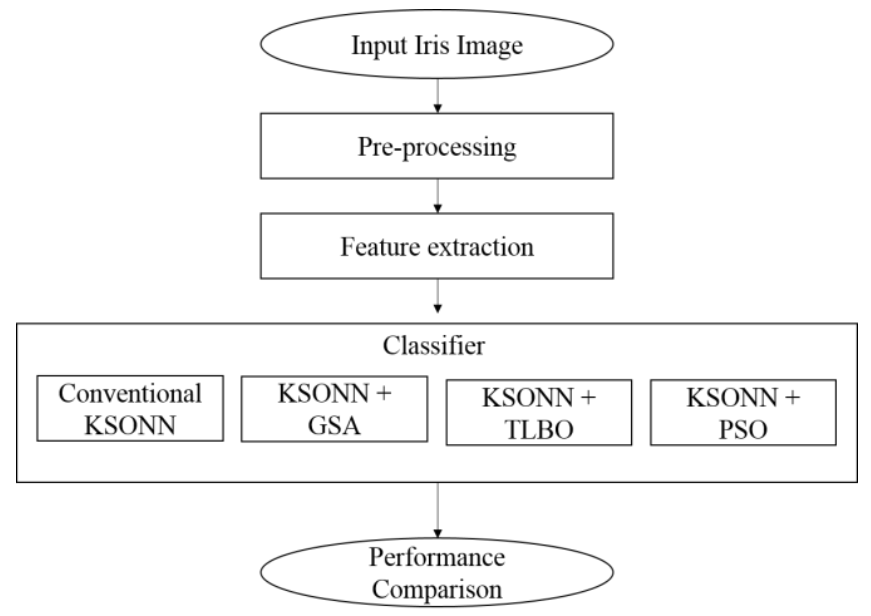

Figure 2. Outline of the proposed methodology

The figure 2 outlines the proposed methodology. Here, in this paper, Gravity Search Algorithm - a physics based method and Particle Swarm Optimization - a swarm intelligence method is adopted into the KSONN to improve the performance of the KSONN classifier. The images for testing the proposed methodology are taken from the IIT-Delhi database.

\section{Conventional Kohonen Self-Organizing Network}

The conventional Kohonen Self-Organizing Network [13], is a simple two-layer artificial neural network. The input layer consists of 1-dimensional neurons and the output layer 
consists of 2-dimensional neurons. The architecture of the same is shown in figure 3. It is an example of an unsupervised classifier in machine learning.

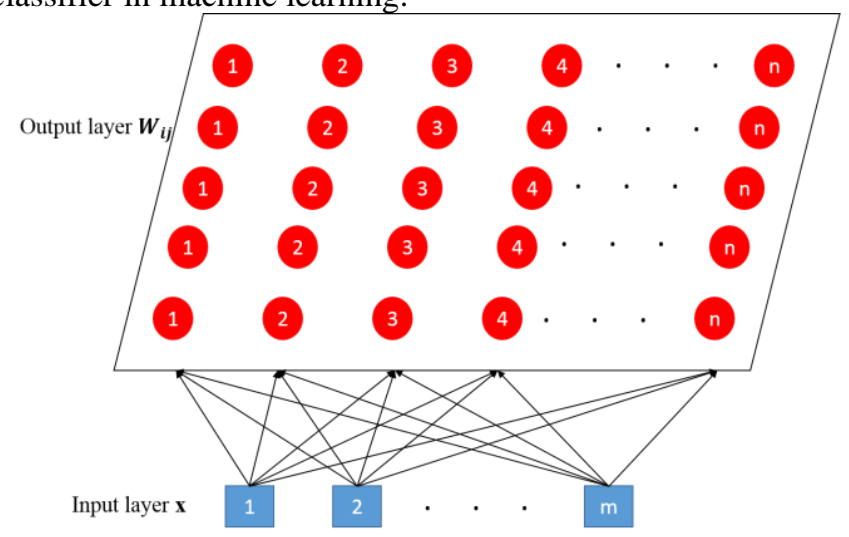

Figure 3. Architecture of KSONN

The feature vectors of the iris are fed to the input layer of the network. The output layer is initialized with random weights. The network finds the output node close to the input vector as the winning node. The weights of the winning node are renewed with the equation (1).

$W_{k}=W_{k-1}+\alpha_{r}\left(x-W_{k-1}\right)$

This process repeats for a maximum number of iterations such as the network has learned the input vectors of all the class. The sequential steps involved in the

Step (1): Random initialization of output weight vectors.

Step (2): Find the Euclidean distance between the input vector and all the output nodes.

Step (3): The node with minimum distance is assigned as winner node.

Step (4): The weights of the winner node are updated using equation (1).

Step (5): This procedure is repeated from step 2 for maximum number of iterations.

Thus, Kohonen learning process for a simple artificial neural network is achieved. This method of learning is termed as unsupervised learning.

\section{Hybrid Kohonen Self-Organizing Neural Network}

The conventional Kohonen Self-Organizing Neural Network has a slow learning phase because of the learning coefficient. In some cases, this may not result in the needed accuracy for implementing a reliable real-time system. Hence, we adopt optimization techniques into the learning phase to improve the performance of the iris recognition system. Here in this study, Gravity Search Algorithm (GSA), Teaching Learning Based Optimization (TLBO) and Particle Swarm Optimization (PSO) is adopted for optimization. A comparison of these methods is also shown to show its robustness.

\subsection{Hybrid Kohonen Self-Organizing Neural Network with GSA}

The cyclic process involved in the learning phase of KSONN is improvised by the inclusion of a physics based optimization technique. Here, Gravity Search Algorithm [14] is adopted into the KSONN. From the randomly initialized output nodes, the GSA optimization helps the KSONN to settle to the feature vector. This method involves the following steps.

Step (1): Random initialization of output weight vectors.

Step (2): Assign the maximum iteration count, gravity constant $G=100$, mass $M=1$, constant $\alpha=2$.

Step (3): Find the Euclidean distance between the input feature vector and all the output nodes. This shows the similarity of the input vector with the output node.

Step (4): The node with the minimum distance is assigned as winner node.

Step (5): The changing gravity constant

$$
\mathrm{G}_{\mathrm{t}}=\mathrm{Ge}^{-\alpha \mathrm{t}}
$$

Step (6): The force on the winning node is give as

$$
\mathrm{F}=\mathrm{G}_{\mathrm{t}} \cdot \mathrm{M} \cdot \delta^{-1} \cdot\left(\mathrm{W}_{\mathrm{j}}-\mathrm{W}_{\mathrm{k}-1}(:, \mathrm{j})\right)
$$

Step (7): The acceleration of the particle is computed by the Newton's law of force,

$$
\mathrm{F}=\mathrm{M} \cdot \mathrm{a}
$$

Step (8): The weights are updated based on the equations (5) The updated position,

$$
W_{k}=W_{k-1}+a
$$

Step (9): Based on the value of the cost function update the $\mathrm{W}_{\text {best. }}$

Step (10): Check whether the maximum is reached. If not keep repeating from step 3 .

Thus, the Newton's law of gravity and motion helps the KSONN network here to settle to the feature vector in the cyclic process.

\subsection{Hybrid Kohonen Self-Organizing Neural Network with TLBO}

Inspired by the human perspective of optimizing the problem, here a Teaching Learning Based Optimization [15] is adopted into the KSONN network. This method involves the following steps.

Step (1): Random initialization of output weight vectors. 
Step (2): Assign the maximum iteration count and best values $\mathrm{W}_{\text {best. }}$.

Step (3): Find the Euclidean distance between the input feature vector and all the output nodes. This shows the similarity of the input vector with the output node.

Step (4): The node with the minimum distance is assigned as winner node $-\mathrm{j}$.

Step (5): Particle position based on teaching factor, $\mathrm{W}_{\mathrm{tl}}=\mathrm{W}_{\mathrm{t}-1}+\operatorname{rand}_{1} \cdot\left(\mathrm{W}_{\mathrm{t}-1}-\mathrm{t}_{\mathrm{f}} \cdot\right.$ mean $)$

Step (6): Update the $\mathrm{W}_{\text {best }}$ based on the cost of the fitness function of $\mathrm{W}_{\mathrm{tl}}$.

Step (7): New weight vector proposed is given as,

$$
\mathrm{W}_{\mathrm{p}}=\mathrm{W}_{\text {best }}+\text { rand }_{1}
$$

Step (8): Learned weight vector is updated based on the cost of the fitness function $\mathrm{W}_{\mathrm{p}}$ and $\mathrm{W}_{\mathrm{tl}}$.

$$
\mathrm{W}_{\mathrm{lr}}=\mathrm{W}_{\mathrm{t}-1}+\operatorname{rand}_{1} \cdot\left(\mathrm{W}_{\mathrm{p}}-\mathrm{W}_{\mathrm{tl}}\right)
$$

Step (9): Update the $W_{\text {best }}$ based on the cost of the fitness of function of $\mathrm{W}_{\mathrm{lr}}$.

Step (10): Check whether the maximum iteration is reached. If not repeat from step 3 .

\subsection{Hybrid Kohonen Self-Organizing Neural Network with PSO}

Into the conventional Kohonen Self-Organizing Neural Network, a swarm intelligence based optimization is introduced. Here, Particle Swarm Optimization [16] is adopted to help the KSONN in the learning phase. It aids the output node to settle to the respective feature vector. This method involves the following steps.

Step (1): Random initialization of output weight vectors.

Step (2): Assign PSO constants $\mathrm{K}_{1}, \mathrm{~K}_{2}, \mathrm{~K}_{\mathrm{i}}, \mathrm{K}_{\mathrm{d}}, \mathrm{d}_{1}$, and $\mathrm{d}_{2}$ with random values.

Step (3): Find the Euclidean distance between the input feature vector and all the output nodes.

Step (4): The node with the minimum distance is assigned as winner node.

Step (5): The velocity for the particle is given by,

$$
\mathrm{v}_{\mathrm{t}}=\mathrm{K}_{\mathrm{i}} \cdot \mathrm{v}_{\mathrm{t}-1}+\mathrm{K}_{1}\left(\mathrm{p}_{\text {best }}-\mathrm{W}_{\mathrm{t}-1}\right)+\mathrm{K}_{2}\left(\mathrm{~g}_{\text {best }}-\mathrm{W}_{\mathrm{t}-1}\right)
$$

Step (6): The weights are updated based on equation (10). The updated position,

$W_{t}=W_{t-1}+v_{t}$

Step (7): Based on the cost of the fitness value update the personal best $p_{\text {best }}$ and global best $g_{\text {best }}$.
Step (8): Update the constant $\mathrm{K}_{\mathrm{i}}$ using the below equation.

$$
\mathrm{K}_{\mathrm{i}}=\mathrm{K}_{\mathrm{i}} \cdot \mathrm{K}_{\mathrm{d}}
$$

Step (9): Replace the $\mathrm{W}_{\mathrm{t}}$ with $\mathrm{g}_{\text {best. }}$

Step (10): Check whether the maximum is reached. If not keep repeating from step 3 .

This process in more pivotal in the learning phase of the KSONN, as the weights are properly directed with the help of personal best and global best to converge with the solution in the vector space.

\section{Results and discussion}

The results of the experiments conducted on the publically available database are presented in this section. It briefs about the specificities of the iris database and indicators for evaluating the proposed method. Later a comparison and analysis of the proposed method with other methods are shown to demonstrate the steadiness of the proposed method.

\subsection{Database}

Iris images are downloaded from the IIT-Delhi database. These images are captured in near infrared spectrum using JIRIS, JPC1000 digital CMOS camera under an indoor environment. It consists of iris images in bitmap format with a resolution of $320 \times 240$ pixels. It holds around 1120 images acquired from 224 subjects. Figure 4 shows a few samples of iris images from the iris database.
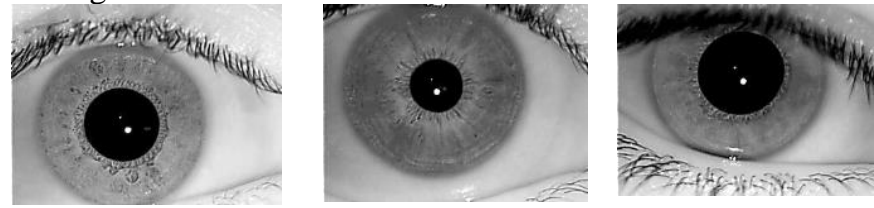

Figure 4. Sample iris images from IIT-Delhi database

The dataset is divided into training set and testing set. The images under training set are used by the network to learn and the images under testing set are used to verify the proposed method. All the simulations are carried on using MATLAB $2017 \mathrm{~b}$ set up on Intel i5 processor with $1.7 \mathrm{GHz}$ clock rate and 4 GB of RAM.

\subsection{Performance metrics}

The proposed method is compared with the conventional KSONN. The performance of all the classifiers are evaluated using the following metrics, a) accuracy, b) specificity, c) sensitivity, d) error rate and e) number of iterations.

Accuracy - Accuracy gives the percentage of matches with the database that is true.

Specificity - Specificity is also called true negative rate. It gives the measure of the classifier identifying the true negatives. 
Sensitivity - Sensitivity is also called true positive rate. It gives the measure of the classifier identifying the true positives.

Error rate - Error rate is the rate of misclassification.

All these metrics are calculated with the confusion matrix created with the results obtained after simulations.

Number of iterations - Iteration is the count of number of times, a process gets repeated to learn the weights.

The iterations define the speed in the learning process. If the number of iterations is large, the network takes more time to converge. Similarly, if the number of iterations is less, the network learns fast to converge.

\subsection{Comparative analysis}

Results are averaged over the five simulations. Table 1 shows the comparison of the performance metrics of all the methods. It can be seen that conventional KSONN shows an accuracy of just $84.33 \%$ and make it impracticable. This disadvantage is nullified by the adoption of optimization method into the KSONN architecture.

Table 1. Accuracy Measures of the classifiers

\begin{tabular}{|c|c|c|c|c|}
\hline Method & $\begin{array}{c}\text { Accurac } \\
\mathbf{y}\end{array}$ & $\begin{array}{c}\text { Speci } \\
\text { ficity }\end{array}$ & $\begin{array}{c}\text { Sensi } \\
\text { tivity }\end{array}$ & $\begin{array}{c}\text { Error } \\
\text { rate }\end{array}$ \\
\hline $\begin{array}{c}\text { Conventional } \\
\text { KSONN }\end{array}$ & $84.33 \%$ & 90.7 & 51 & $15.67 \%$ \\
\hline KSONN+GSA & $94.8 \%$ & 97.01 & 78 & $5.2 \%$ \\
\hline KSON+TLBO & $96.2 \%$ & 97.85 & 83.33 & $3.8 \%$ \\
\hline KSONN+PSO & $\mathbf{9 8 \%}$ & $\mathbf{9 9 . 0 6}$ & $\mathbf{9 2}$ & $\mathbf{2 \%}$ \\
\hline
\end{tabular}

The Newtonian law of GSA gives a fair improvement in the performance of KSONN. The force and acceleration of the weights in the learning process help in the proper identification of the weights in the search space. However, the acceleration of the process causes some uncertainty in the converged values. As a result, this method achieves an accuracy of $94.8 \%$.

The human-inspired TLBO gives a relatively better performance for KSONN. The psychology between a teacher and learner is used to explore the weights in the search space. This method achieves an accuracy of $96.2 \%$.

The Swarm intelligence method of optimization by PSO gives a better performance for the KSONN network. Here the personal best and global best give proper directions during the exploration process to learn the weights from the search space. As it has multiple directors, the learning process is effective. This method achieves an accuracy of $98 \%$.

From the comparative analysis made, it is evident that the inclusion of the optimization technique into the KSONN has improved the accuracy. The PSO, a swarm-based optimization performs compared to the other two optimization methods. The error rate is also minimized.

Apart from increasing the accuracy of the iris recognition, the optimization techniques also reduce the iterations needed for the network to learn.

Table 2. Convergence Analysis of the classifiers

\begin{tabular}{|c|c|}
\hline Method & No. of iterations required \\
\hline Conventional KSONN & 250 \\
\hline KSONN+GSA & 120 \\
\hline KSON+TLBO & 92 \\
\hline KSONN+PSO & $\mathbf{8 5}$ \\
\hline
\end{tabular}

Table 2 provides a comparison of iterations consumed by different methods. The iterations are controlled by the learning rate. If the learning rate is low, the network takes more iterations in the learning phase. However, a fast learning rate cannot guarantee better convergence. The optimization method helps to achieve a descent learning rate to identify the best results. The conventional KSONN, has a very low learning rate hence it takes more iterations to converge. It takes around 250 iterations in this simulation. KSONN with GSA takes 120 iterations, KSONN with TLBO takes 92 iterations and KSONN with PSO takes 85 iterations to converge.

\section{Conclusion}

This paper has reported on combining of optimization techniques with the KSONN architecture. Form the experiments conducted on IIT-Delhi iris database, conventional KSONN procures an accuracy of $84.3 \%$, KSONN with GSA achieves an accuracy of $94.8 \%$, KSONN with TLBO achieves an accuracy of $96.2 \%$ and KSONN with PSO achieves an accuracy of $98 \%$. Moreover, the iteration took in the learning process of conventional KSONN of 250 , KSONN with GSA took 120, KSONN with TLBO took 92 and KSONN with PSO took 85. Out of the optimization techniques applied, swarm intelligence-based optimization has resulted in enhanced performance.

These approaches are normally used to identify the culprits in crime detection applications. Since the iris of each human being is unique, a database of the iris of all the criminals can be created. All the crimes/cases associated with a specific criminal can be linked with the iris images. A matching process can be used to determine the previous offenses of a specific culprit.

\section{References}

[1] John Daugman. "How Iris Recognition Works". IEEE Transactions on Circuits and Systems for Video Technology, volume - 14, pp. 21-30, (2004). 
[2] Richard P. Wildes. "Iris Recognition: An Emerging Biometric Technology". Proceedings of IEEE, Volume85, pp. 1348-1363, (1997).

[3] P. Radu, K. Sirlantzis, G. Howells, S. Hoque and F. Deravi. "A Color Iris Recognition System Employing Multiple Classifier Techniques". Electronics Letters on Computer Vision and Image Analysis, Volume-12(2), pp. 54-65, (2013)

[4] Md. Rabiul Islam. "Feature and Score Fusion Based Multiple Classifier Selection for Iris Recognition". Computational Intelligence and Neuroscience, Volume2014, pp. 1-11, (2014).

[5] C. Rathgeb, F. Breitinger, C. Busch and H. Baier. "On application of bloom filters to iris biometrics". IET Biometrics, Volume-3(4), pp. 207-218, (2014).

[6] V.V.S. Tallapragada and E.G. Rajan. "Morphology Based Non-Ideal Iris Recognition using Decision Tree Classifier". International Conference on Pervasive Computing, pp. 1-4, (2015).

[7] Y. Si, J. Mei and H. Gao. "Novel Approaches to Improve Robustness, Accuracy and Rapidity of Iris Recognition System". IEEE Transactions on Industrial Informatics, Volume-8(1), pp. 110-117, (2012).

[8] Y. Song, W. Cao and Z. He. "Robust iris recognition using error correction model and discriminative dictionary learning". Neurocomputing, Volume-137(5), pp. 198-204, (2014).

[9] V.V.S. Tallapragada and E.G. Rajan. "Improved kernelbased IRIS recognition system in the framework of support vector machine and hidden Markov model". IET Image Processing, Volume-6(6), pp. 661-667, (2012).

[10] R.M. Farouk, R. Kumar and K.A. Riad. "Iris matching using multi-dimensional artificial neural network". IET Computer Vision, Volume-5(3), pp. 178-184, (2011).

[11] D. Choudhary, A. K. Singh and Shamik Tiwari. "A Statistical Approach for Iris Recognition Using K-NN Classifier". International Journal of Image, Graphics and Signal Processing, Volume-2013(4), pp. 46-52, (2013).

[12] M.Z. Rashad, M.Y. Shams, O. Nomir and R.M. ElAwady. "Iris Recognition Based on LBP and Combined LVQ Classifier". International Journal of Computer Science \& Information Technology, Volume-3(5), pp. 67-78, (2011).

[13] T. Kohonen. "The Self-Organizing Map". Proceedings of IEEE, Volume-78(9), pp. 1464-1480, (1990).

[14] E. Rashedi, H. Nezambadi-pour and S. Saryazdi. "GSA: A Gravitational Search Algorithm". Information Sciences, Volume:179(2009), pp. 2232-2248, (2009).

[15] J. Kennedy, R. Eberhart. "Particle Swarm Optimization". Proceedings of International Conference on Neural Networks, pp. 1942-1948, (1995).

[16] R.V. Rao, V.J. Savasani and D.P. Vakharia. "TeachingLearning-Based Optimization: An optimization method for continuous non-linear scale problems". Information Sciences, volume-183(2012), pp. 1-15, (2012). 